\title{
Assessment of reaching proficiency in procedural skills: fiberoptic airway simulator training in novices
}

This article was published in the following Dove Press journal:

Open Access Medical Statistics

17 November 201।

Number of times this article has been viewed

\author{
Xinyuan Duan' \\ Dongfeng $\mathrm{Wu}^{\prime}$ \\ Alexander F Bautista ${ }^{2}$ \\ Ozan Akca ${ }^{2}$ \\ Mary B Carter ${ }^{3}$ \\ Rana K Latif ${ }^{2}$ \\ 'Department of Bioinformatics and \\ Biostatistics, School of Public Health \\ and Information Sciences, University \\ of Louisville, Louisville, KY, USA; \\ ${ }^{2}$ Paris Simulation Center, Department \\ of Anesthesiology and Perioperative \\ Medicine, University of Louisville \\ School of Medicine, Louisville, \\ KY, USA and Outcomes Research \\ Consortium, ${ }^{3}$ Medical Education \\ Research Unit, Office of Medical \\ Education, University of Louisville \\ School of Medicine, Louisville, \\ KY, USA
}

Background: The importance of fiberoptic bronchoscopy (FOB) and intubation (FOI) is well established to reduce anesthesia-related morbidity and mortality during airway management in clinical practice. We conducted a trial to determine whether simulation-based training of novices can increase FOB and FOI skills to an expert level.

Methods: Eight anesthesiologists as experts and 15 fourth-year medical students as novices were enrolled. The computerized AccuTouch ${ }^{\circledR}$ Bronchoscopy Simulator (ATBS, Immersion Medical, Gaithersburg, MD) and the Human Airway Anatomy Simulator (HAAS, Medical Plastics Inc, Gatesville, TX) were used for FOB and FOI training and testing, respectively. Data included three discrete variables: the numbers of airway collisions, oral passes, and nasal passes. Experts were tested, while novices were tested, trained, and retested. Twelve novices returned after 2 months and were again tested, retrained, and retested. Data were analyzed by a newly proposed likelihood ratio test based on the assumption that the data follow a Poisson distribution. A testing procedure was derived to compare performance between experts, novices pre- and post-training, and novices after the 2-month lag period.

Results: As expected, experts outperformed pre-trained novices on all variables $(P<0.0001)$. Post-training, novices' skills improved $(P<0.0001)$ to the point that there were no significant differences between experts and novices post-training $(P=0.1709,0.9857$, and 0.5014 for collisions, and oral and nasal passes, respectively). After 2 months there was a significant decay in FOB skills among novices compared to the prior level achieved $(P<0.0001)$, but brief retraining brought their performance back to the expert level $(P=0.2207)$.

Conclusion: Due to the small sample size, normal approximation is not applicable; therefore many existing tests are not appropriate. Our novel likelihood ratio test provided a powerful tool in analyzing the small samples of discrete training data. Simulation can be used to train novices in fiberoptic airway skills to an expert level in a relatively short period of time.

Keywords: fiberoptic intubation skills, simulator, medical simulation, medical education, maximum likelihood estimate, likelihood ratio test

\section{Introduction}

Mismanagement of the difficult airway remains the most common cause of anesthesiarelated morbidity and mortality. ${ }^{1}$ The importance of fiberoptic intubation (FOI), whether via the oral or nasal approach, is well established in the management of the difficult airway, ${ }^{2-4}$ but recent surveys revealed that the prevalence of FOI skills is still lacking among anesthesiologists. ${ }^{5-8}$ Fiberoptic bronchoscopy (FOB) is another skill closely related to FOI. Both FOB and FOI involve introducing a fiberoptic scope into the airway of a breathing patient. Care must be taken to avoid collisions with the walls
Correspondence: Rana K Latif Department of Anesthesiology and Perioperative Medicine, University of Louisville School of Medicine, Louisville, Kentucky 40202, USA

Tel + I 502852585 I

Email rklati0I@louisville.edu
(C) 20 I I Duan et al, publisher and licensee Dove Medical Press Ltd. This is an Open Access article which permits unrestricted noncommercial use, provided the original work is properly cited. 
of the pharynx, larynx, trachea, and bronchi. Adequate skill must be attained to ensure successful intubation on the first pass of the bronchoscope. The role of simulation in training novices in FOB and FOI is as yet under-explored.

Studies show that repetitive practice is required to maintain skill level ${ }^{9}$ after training, and so skill decay may occur during periods of little or no exposure. Once trained to the 'expert' level, the extent of skill decay over time among novices has not been studied. As residents spend less time in operating rooms, ${ }^{10}$ there is a clear need to accelerate the learning curve and improve the efficacy of FOB and FOI skills training. Furthermore, although novice practitioners are the reality of the academic care environment, patient care should preferably be provided by novices with as much previous training as possible under attending physician supervision.

We planned an educational trial to determine whether a 1.5 hour episode of simulation based training of novices can increase FOB and FOI skills to an expert level, and also to assess the extent of decay over a time period of 2 months. However, since the data collected were discrete rather than continuous variables, and sample sizes were small ( 8 and 15 , respectively), standard $t$-test or analysis of variance (ANOVA) methodology would not be appropriate to use in this case. Both methods assume that either the data come from an approximately normal distribution, or the sample size is fairly large (for example, $n>20$ at the very least). We sought a better statistical method to overcome these difficulties. The purpose of this study is to present a newly proposed likelihood ratio test to analyze discrete fiberoptic airway skill data. The development of this model was based on the assumption that the data follow a Poisson distribution.

\section{Materials and methods}

This study was approved by our Institutional Review Board. We utilized two simulators for training and testing: (1) the computerized AccuTouch ${ }^{\circledR}$ Bronchoscopy Simulator (ATBS, Immersion Medical, Gaithersburg, MD) for FOB skills, and (2) the Human Airway Anatomy Simulator (HAAS, Medical Plastics Inc, Gatesville, TX) for FOI skills. With the ATBS, the number of airway collisions (contacts between the fiberoptic bronchoscope and the inside wall of the tracheobronchial tree) are tallied and reported by the computer. Greater expertise is demonstrated by a fewer number of collisions. Using the HAAS, FOI may occur via either an oral or nasal approach, and the fewer number of passes (attempts) the more expert the operator. Because the HAAS is not computerized, the number of oral and nasal passes must be quantified by an observer. For this study, subjects were videotaped with their identity concealed while performing FOI. A blinded, trained observer reviewed the recordings and tallied the number of oral and nasal passes of each participant.

\section{Novice and expert groups}

We recruited eight faculty anesthesiologists who had performed more than 100 fiberoptic intubations apiece as the expert group and 15 fourth-year medical students who had never performed a fiberoptic intubation as the novice group. Experts underwent testing only and were asked to perform FOB and both oral and nasal FOI on the ATBS and HAAS simulators, respectively. Novices underwent testing followed by a 1.5 -hour simulation-based training module and immediate retesting. For each subject, the number of airway collisions during FOB was recorded by the ATBS computer while the number of oral and nasal passes during FOI was recorded by the blinded expert after reviewing all videotaped recordings. Two months after the initial training, twelve novices returned for testing, retraining, and retesting in FOB only. The retraining took only 30 minutes.

\section{Statistical methods}

The number of airway collisions during FOB and the number of oral and nasal passes during FOI are discrete variables. A testing procedure based on the likelihood ratio was derived to analyze the difference between the two groups and the change of skill in the novice group both before and after training and over time. The derived test was based on the assumption that the number of airway collisions and oral or nasal passes follows a Poisson distribution.

We will use the number of airway collisions to illustrate. The derivation is as follows: (1) Let the random variable $X_{i}$ represent the number of airway collisions of subject $i$ in the expert group $(i=1,2, \ldots, 8)$ and let $\underline{X}=\left(X_{1}, \ldots, X_{8}\right)$ be the random vector; (2) let random variable $Y_{j}$ represent the number of airway collisions for subject $\mathrm{j}$ in the novice group pre-training $(j=1,2, \ldots, 15)$ and thus $\underline{Y}=\left(Y_{1}, \ldots, Y_{15}\right)$ is the associated random vector. The likelihood function is: $L(\lambda \mid \underline{X})=\Pi_{i=1}^{n} e^{-\lambda} \lambda^{x_{i}} / x_{i} !$, and therefore, the Maximum Likelihood Estimate (MLE) is: $\hat{\lambda}_{1}=1 / n \sum_{i=1}^{n} X_{i}$ for the expert group; and $\hat{\lambda}_{2}=1 / m \sum_{j=1}^{m} Y_{j}$ for the novice group, where the sample sizes are $n=8$ and $m=15$, respectively. It is also known that $\sum_{i=1}^{n} X_{i}$ follows a Poisson distribution with mean $n \lambda_{1}$ and $\sum_{j=1}^{m} Y_{j}$ follows a Poisson distribution with mean $m \lambda_{2}$. 
To compare the parameters $\lambda_{1}$ and $\lambda_{2}$, the average number of collisions in the expert and novice groups, the null and the alternative hypotheses will be:

$$
H_{0}: \lambda_{1}=\lambda_{2} \text { versus } H_{a}: \lambda_{2}>\lambda_{1} .
$$

Letting $\lambda=\lambda_{1}$ and $\Phi=\lambda_{2} / \lambda_{1}$, the hypotheses are transformed to:

$$
H_{0}: \Phi=1 \text { versus } H_{a}: \Phi>1 .
$$

Thus $X_{i}$ follows a Poisson distribution with parameter $\lambda$, and $Y_{j}$ follows a Poisson distribution with parameter $\lambda \Phi$. If we let $S_{X}=\sum_{i=1}^{n} X_{i}$ and $S_{Y}=\sum_{j=1}^{m} Y_{j}$, then $S_{0}=S_{X}+S_{Y}$ follows a Poisson distribution with parameter $n \lambda+m \lambda \Phi$, and the conditional Probability Mass Function (PMF) of $S_{Y}$ given $S_{0}$ will be:

$$
\begin{aligned}
P & \left(S_{Y}=s_{2} \mid S_{0}=s_{0}, \lambda, \Phi\right) \\
& =\frac{P\left(S_{X}=s_{1}, S_{Y}=s_{2} \mid \lambda, \Phi\right)}{P\left(S_{0}=s_{0} \mid \lambda, \Phi\right)} \\
& =\left(\begin{array}{l}
s_{0} \\
s_{2}
\end{array}\right)\left(\frac{n}{n+m \Phi}\right)^{s_{1}}\left(\frac{m \Phi}{n+m \Phi}\right)^{s_{2}} \text { if } s_{1}+s_{2}=s_{0} .
\end{aligned}
$$

Hence the conditional distribution of $S_{Y}$ given $S_{0}$ follows a binomialdistribution with parameters $\left(S_{0}, p=m \Phi /(n+m \Phi)\right)$, and the hypothesis testing becomes:

$H_{0}: p=\frac{m}{m+n}($ if $\Phi=1)$ versus

$H_{a}: p>\frac{m}{m+n}($ if $\Phi>1)$.
To compare the performance of novices pre- and post-training, or after the 2-month lag period, we used the following $P$ value equation:

$$
\begin{aligned}
& P \text {-value }=P\left(S_{Y} \geq S_{Y} \mid S_{X}+S_{Y}=S_{0}, \Phi=1\right) \\
& \quad=P\left(S_{Y} \geq S_{Y} \mid p=\frac{m}{m+n}\right) \\
& =\sum_{K=S_{Y}}^{S_{0}}\left(\begin{array}{l}
S_{0} \\
K
\end{array}\right) p^{K}(1-p)^{S_{0}-K}
\end{aligned}
$$

To compare the performance of the novice group posttraining or after the 2-month lag period to the expert group, we used the following normal approximation, as sample size $S_{0}$ is larger than 20 for a two-sided test:

$$
P=2 \times P(Z \geq|z|)=2 \times p\left(Z>\left|\frac{S_{Y}-p S_{0}}{\sqrt{S_{0} p q}}\right|\right)
$$

\section{Results}

We developed a novel likelihood ratio test to analyze the simulation training results. For the number of airway collisions during FOB, we compared the pre-training and post-training performances of novices, and also compared the pre- and post-training performances of novices to that of experts. Before training, the number of airway collisions in the novice group was significantly greater than in the expert group $(P<0.0001)$ indicating decreased skill, but their performance improved significantly after the initial 1.5 -hour training session $(P<0.0001$, novices pre- vs post-training $)$ to the expert

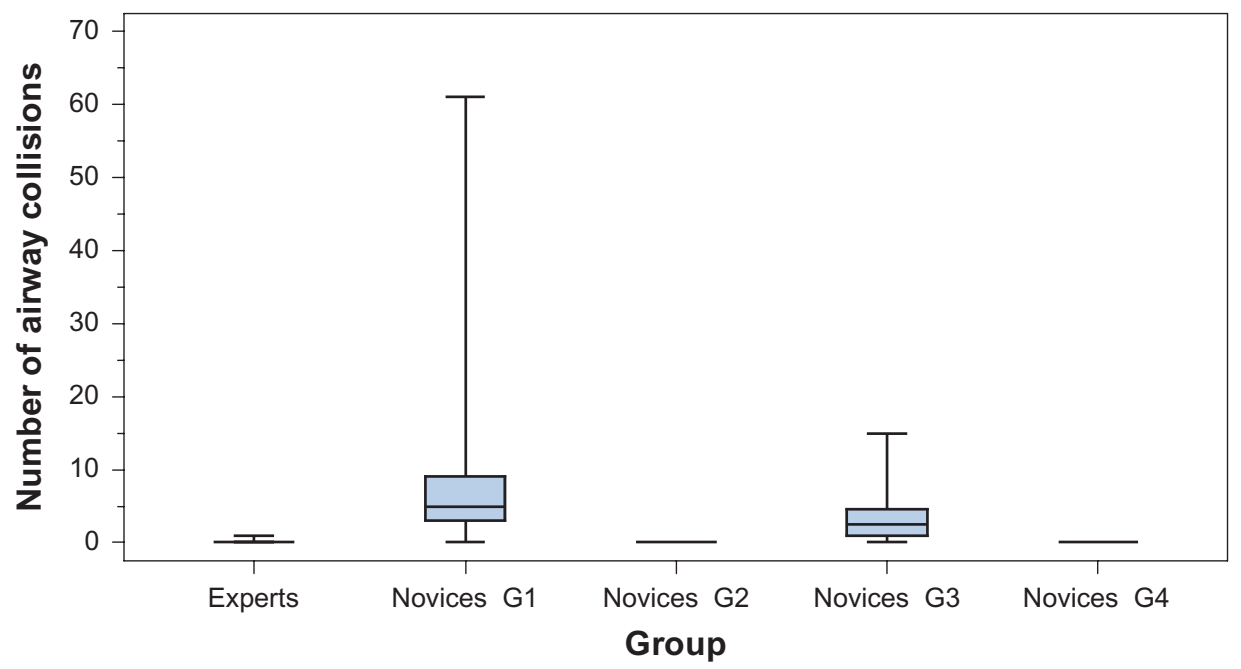

Figure I Box-plot of the number of airway collisions in each group during fiberoptic bronchoscopy using the AccuTouch ${ }^{\circledR}$ Bronchoscopy Simulator (with $95 \%$ confidence interval). Expertise is demonstrated by fewer collisions. The minimum number of collisions possible is zero.

Abbreviations: Novices GI, Novice group pre-training; Novices G2, Novice group post-training; Novices G3, Novice group 2 months later before retraining; Novices G4, Novice group 2 months later after retraining. 
Table I $P$ values resulting from the comparisons of the number of airway collisions in each group during fiberoptic bronchoscopy using the AccuTouch ${ }^{\circledR}$ Bronchoscopy Simulator. (A) novices versus experts; (B) novices pre- and post-training versus novices 2 months later

A

\begin{tabular}{|c|c|c|c|c|}
\hline & \multicolumn{2}{|l|}{ Novices } & \multirow{2}{*}{$\frac{\text { Novices }}{\text { Before retraining }}$} & \multirow{2}{*}{$\begin{array}{l}\mathbf{2} \text { months later } \\
\text { after retraining }\end{array}$} \\
\hline & Pre-training & Post-training & & \\
\hline Experts & $<0.0001$ & 0.1709 & $<0.0001$ & 0.2207 \\
\hline \multicolumn{5}{|l|}{ B } \\
\hline \multirow{2}{*}{$\begin{array}{l}\text { Novices } \\
2 \text { months later }\end{array}$} & \multicolumn{2}{|l|}{ Novices } & & \\
\hline & Pre-training & Post-training & & \\
\hline Before retraining & $<0.0001$ & $<0.0001$ & & \\
\hline After retraining & $<0.0001$ & Close to I & & \\
\hline
\end{tabular}

level $(P=0.1709$, novices post-training vs experts, Figure 1 , Table 1). Newly acquired skills of novices decayed significantly after 2 months $(P<0.0001$, novices post-training vs novices 2 months later before retraining). Despite this decay, skills remained statistically greater than the pre-training level
( $P<0.0001$, novices pre-training vs novices 2 months later before retraining). Although skills decayed over time, the previously trained novice was easily retrained to the expert level of FOB after only a 30-minute refresher course $(P=0.2207$, novices after retraining vs experts).

A

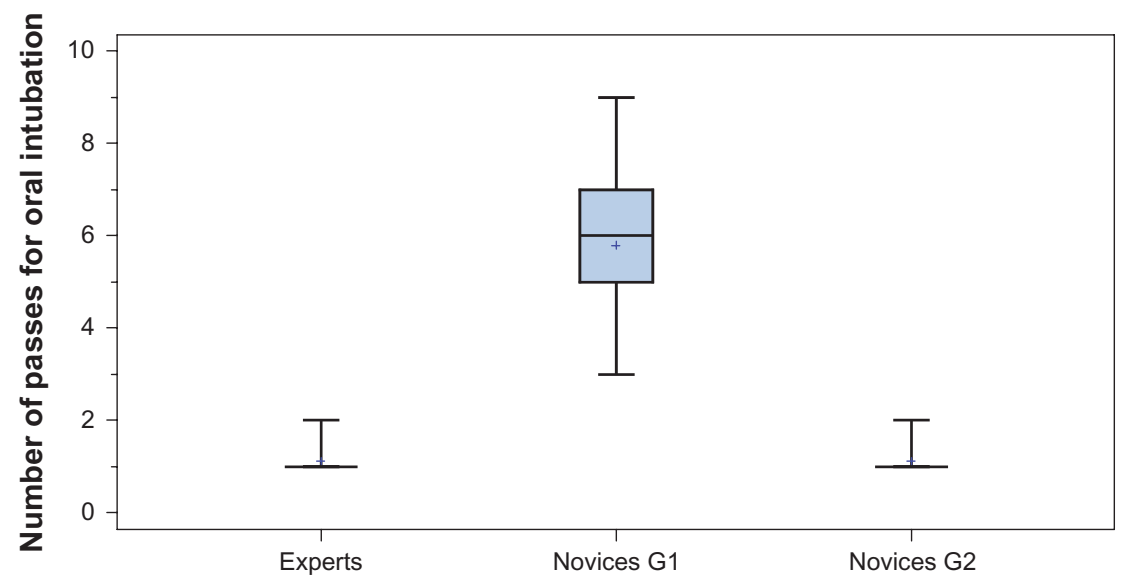

Group

B

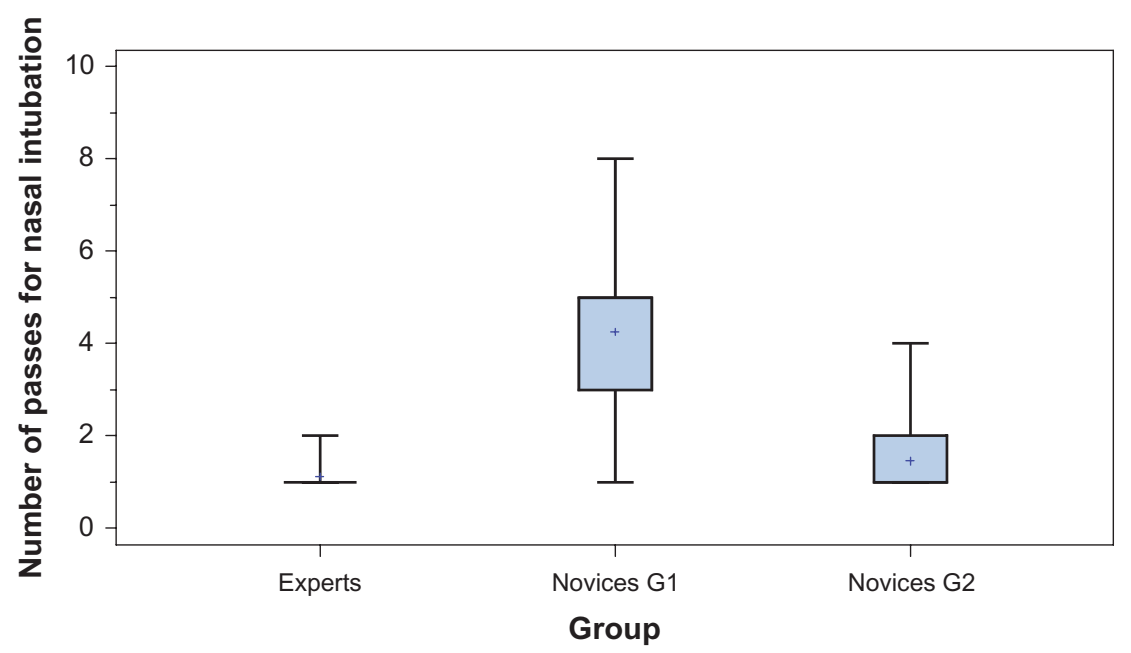

Figure 2 Box-plot of the number of passes in each group for oral (A) and nasal (B) fiberoptic intubation using the Human Airway Anatomy Simulator (with $95 \%$ confidence interval). Expertise is demonstrated by fewer passes (attempts). The minimum number of passes possible is one.

Abbreviations: Novices GI, Novice group pre-training; Novices G2, Novice group post-training. 
Likewise, before training, the number of passes for oral and nasal FOI in the novice group was significantly greater than in the expert group $(P<0.0001$ for both oral and nasal, novices pre-training vs experts, Figure 2, Table 2) indicating decreased skill among novices before training. FOI skill increased significantly after the initial 1.5 -hour training session $(P<0.0001$ for both oral and nasal, novices pre- vs post-training) until reaching the expert level (oral: $P=0.9857$; nasal: $P=0.5014$; novices post-training vs experts).

\section{Discussion and conclusion}

In this study, we present a new likelihood ratio test that we developed to analyze fiberoptic skills after simulation-based training. The development of this model was based on the assumption that the data follow a Poisson distribution. This method can be used for analyzing other similar discrete data, especially with a small sample size, when the commonly used $t$-test or ANOVA cannot be applied. The analysis of our fiberoptic bronchoscopy and fiberoptic intubation data demonstrates the efficacy of our novel likelihood ratio test.

Selecting the correct methodology for evaluating bronchoscopic simulator data can be a challenge. Davoudi et $\mathrm{al}^{11}$ used analysis of variance (ANOVA) and pairwise differences with repeated measures ANOVA to analyze data from a virtual reality bronchoscopic simulator. However, to use ANOVA, the underlying assumption is that either the data come from a normal distribution, or the sample size is large. In the current simulator study, these two assumptions were not satisfied, and therefore, there was a need to develop a new statistical test.

Our results showed that the novice group achieved an expert level of performance on the simulator in terms of number of airway collisions, and number of oral and nasal passes. Although skill among novices decayed over a 2-month period, the decay was not total and skill remained greater than pre-training levels. A brief retraining session successfully restored FOB skill to the expert level. Admittedly, achieving superior skill on a simulator does

Table $2 P$ values resulting from the comparisons of the number of passes (attempts) for oral and nasal fiberoptic intubation in each group using the Human Airway Anatomy Simulator

\begin{tabular}{llll}
\hline & $\begin{array}{l}\text { Novices } \\
\text { pre-training vs } \\
\text { post-training }\end{array}$ & $\begin{array}{l}\text { Novices } \\
\text { pre-training vs } \\
\text { experts }\end{array}$ & $\begin{array}{l}\text { Novices } \\
\text { post-training } \\
\text { vs experts }\end{array}$ \\
\hline Oral & $<0.000 \mathrm{I}$ & $<0.000 \mathrm{I}$ & 0.9857 \\
Nasal & $<0.000 \mathrm{I}$ & $<0.000 \mathrm{I}$ & 0.5014 \\
\hline
\end{tabular}

not automatically indicate expert performance on human patients nor does it guarantee better patient outcomes. Furthermore, how much decay in skill occurs after 4, 6, 8, or even 12 months, and how much retraining is required after these longer lag periods was not examined in this study. Nonetheless, our findings support the claim that simulation-based training may accelerate the learning curve and provide a safe opportunity for novices such as medical students to become more competent in clinical procedures before demonstrating their skill on live patients. Besides the benefit of efficient and accurate training, ${ }^{12,13}$ instruction on the simulator has been shown to enhance learner satisfaction, and reduce the cost of patient care. ${ }^{13,14}$

\section{Acknowledgments}

This study was undertaken with the collaboration of the Paris Simulation Center, Department of Anesthesiology and Perioperative Medicine, and Office of Medical Education at the University of Louisville School of Medicine, and the Outcomes Research Consortium.

\section{Disclosure}

The authors report no conflicts of interest in this work.

\section{References}

1. Kluger MT, Tham EJ, Coleman NA, Runciman WB, Bullock MF Inadequate pre-operative evaluation and preparation: a review of 197 reports from the Australian incident monitoring study. Anaesthesia. 2000;55(12):1173-1178.

2. Practice guidelines for management of the difficult airway. A report by the American Society of Anesthesiologists Task Force on Management of the Difficult Airway. Anesthesiology. 1993;78(3):597-602.

3. Benumof JL. Management of the difficult adult airway. With special emphasis on awake tracheal intubation. Anesthesiology. 1991;75(6): 1087-1110.

4. NCEPOD.org NCEiP-OD. UK: National Confidential Enquiry into Patient Outcome and Death. Available from: www.ncepod.org.uk. Accessed September 27, 2011

5. Popat MT, Srivastava M, Russell R. Awake fibreoptic intubation skills in obstetric patients: a survey of anaesthetists in the Oxford region. Int J Obstet Anesth. 2000;9(2):78-82.

6. Kristensen MS, Møller J. Airway management behaviour, experience and knowledge among Danish anaesthesiologists - room for improvement Acta Anaesthesiologica Scandinavica. 2001;45(9):1181-1185.

7. Rosenstock C, Ostergaard D, Kristensen MS, Lippert A, Ruhnau B, Rasmussen LS. Residents lack knowledge and practical skills in handling the difficult airway. Acta Anaesthesiol Scand. 2004;48(8): 1014-1018.

8. Ezri T, Szmuk P, Warters RD, Katz J, Hagberg CA. Difficult airway management practice patterns among anesthesiologists practicing in the United States: have we made any progress? J Clin Anesth. 2003 15(6):418-422.

9. Arthur Jr W, Bennett W Jr, Stanush PL, McNelly TL. Factors that influence skill decay and retention: a quantitative review and analysis. Human Performance. 1998;11:57-101

10. Tomlinson A. Effects of the EWTD on anaesthetic training in the UK Anaesthesia. 2005;60(1):96-97. 
11. Davoudi M, Osann K, Colt HG. Validation of two instruments to assess technical bronchoscopic skill using virtual reality simulation. Respiration. 2008;76(1):92-101.

12. Naik VN, Natsumoto ED, Houston PL, et al. Fiberoptic orotracheal intubation on anesthetized patients: do manipulation skills learned on a simple model transfer into the operating room? Anesthesiology. 2001; 95(2):343-348.
13. Chen JS, Hsu HH, Lai IR, et al. Validation of a computer-based bronchoscopy simulator developed in Taiwan. J Formos Med Assoc. 2006;105(7):569-576.

14. Davoudi M, Colt HG. Bronchoscopy simulation: a brief review. $A d v$ Health Sci Educ Theory Pract. 2009;14(2):287-296.

\section{Publish your work in this journal}

Open Access Medical Statistics is an international, peer- reviewed, open access journal publishing original research, reports, reviews and commentaries on all areas of medical statistics. The manuscript management system is completely online and includes a very quick and fair

\section{Dovepress}

peer-review system. Visit http://www.dovepress.com/testimonials.php to read real quotes from published authors. 